\title{
A Nullstellensatz and a Positivstellensatz for Ordered Differential Fields
}

\author{
Quentin Brouette*
}

September 25, 2012

\begin{abstract}
We use the model completeness and axiomatisation of the theory of closed ordered differential fields to give a differential version of Dubois' nullstellensatz and Stengle's positivstellensatz for ordered fields.
\end{abstract}

\section{Introduction}

In this paper we extend some results of real geometry and real algebra to the context of ordered differential fields and rings.

Ordered differential fields were studied by M. Singer who showed in [13] that the theory of ordered differential fields has a model completion and called it CODF (acronym for closed ordered differential fields). He gave an axiomatisation of CODF which contains the axioms of the theory RCF of real closed fields and a scheme of axioms expressing that there is a solution to a differential equation whenever there is a solution to the corresponding algebraic equation and such solution can be chosen close to the algebraic one.

Since then one has considered ordered fields with several (commuting) derivations. These fields are called ordered partial differential fields and their model completion is called $m$-CODF (where $m$ is the number of derivations put on the field). M. Tressl and C. Rivière studied this theory and also gave an axiomatisation similar to the case of one derivation (see [16] and [10]).

In the first section, we define the theory CODF and state some relevant well-known results.

Some of our results are motivated by the work of D. W. Dubois (see [3]) on a nullstellensatz for real closed fields (see also [9] and [1] for an other exposition of his results). In section 2, we recall the definition of the real radical of an ideal $I$ and use it to state Dubois' result. We also give some of the necessary background on differential ideals.

In section 3, we prove that if $I$ is a real differential ideal, $I$ is an intersection of prime differential real ideals and we show that the real

*quentin.brouette@gmail.com 
radical of $I$ is the smallest real differential ideal containing $I$. Using the fact that CODF is model complete we prove a nullstellensatz for models of CODF (see Theorem 3.8).

Then in section 4 , we study the behaviour of real differential ideals under restriction. Let $A \subset B$ two differential rings and $I$ be a real differential ideal of $B$. We show that if $I \cap A$ is prime, then $I$ is an intersection of prime differential real ideals and moreover the ideals involved in the intersection can be chosen to have all the same intersection with $A$.

In section 5 , we make use of the fact that in a model of CODF, differential tuples are dense (see Corollary 1.3) in order to transfer a positivstellensatz for models of RCF to models of CODF (see Theorem $5.3)$.

We finally consider partial differential fields, that is, fields endowed with several commuting derivations. In the last section, using T. McGrail and C. Rivière's results (see [7] and [10]) on partial differential fields and ordered partial differential fields, we explain how our results can be generalised to that context.

Since this paper was written, M. Tressl drew our attention to a paper by G. Stengle [15]. In his paper, G. Stengle investigates the real differential spectrum of a differential ring and shows that the real radical of a differential ideal is still differential. He proves a nullstellensatz and a positivstellensatz for CODF as a corollary of an abstract result for differential rings. This abstract theorem (see theorem 4.2 in [15]) was unfortunately proven to be false by $\mathrm{T}$. Grill who gave a counterexample in his Ph.D. thesis ([4], p.47).

\section{Conventions and notations}

Throughout this paper, all rings and fields are commutative and of characteristic zero. A differential ring is a ring $R$ with a derivation, that is a map $R \rightarrow R: x \mapsto x^{\prime}$ such that for all $u, v \in R,(u+v)^{\prime}=u^{\prime}+v^{\prime}$ and $(u v)^{\prime}=u^{\prime} v+u v^{\prime}$. Moreover, we let $u^{(0)}=u$ and $u^{(n+1)}=\left(u^{(n)}\right)^{\prime}$.

Let $E$ be any set and $a_{1}, \ldots, a_{n} \in E$, then $\bar{a}$ will denote the tuple $\left(a_{1}, \ldots, a_{n}\right)$.

For any differential field $F, F\left\{X_{1}, \ldots, X_{n}\right\}$ will denote the differential ring of differential polynomials with coefficients in $F$ i.e. $F\left[X_{i}^{(j)}\right.$ : $i, j \in \mathbb{N}]$. If $L$ is a differential field extension of $F$, for any $A \subset$ $F\left\{X_{1}, \ldots, X_{n}\right\}, \mathcal{V}_{L}(A):=\left\{\bar{a} \in L^{n}: \forall f \in A, f(\bar{a})=0\right\}$ and for any $B \subset L^{n}, \mathcal{I}(B):=\left\{f \in F\left\{X_{1}, \ldots, X_{n}\right\}: \forall \bar{a} \in B, f(\bar{a})=0\right\}$.

Let $f \in F\{X\}$, the order of $f$ is denoted by $\operatorname{ord}(f)$ and is the biggest $k$ such that $X^{(k)}$ appears in $f$. We associate to $f$ an ordinary polynomial $f^{*}$ such that $f(X)=f^{*}\left(X, X^{\prime}, \ldots, X^{(l)}\right)$ and $l$ is minimal. Note that $l=\operatorname{ord}(f)$. Moreover, the separant of $f$ is $s_{f}:=\partial f / \partial X^{(l)}$.

If $f, g \in F\left\{X_{1}, \ldots, X_{n}\right\}$ (or $f, g \in F\left[X_{1}, \ldots, X_{n}\right]$ ) and $W \subset F^{n}$, we will write $f={ }_{W} g$ to say that $\forall \bar{x} \in W, f(\bar{x})=g(\bar{x})$. Note that $f={ }_{W} g$ iff $f-g \in \mathcal{I}(W)$.

If $\bar{u}:=\left(u_{1}, \ldots, u_{n}\right) \in F^{n}$, we note $\bar{u}^{\prime}=\left(u_{1}^{\prime}, \ldots, u_{n}^{\prime}\right)$. Similarly, 
$\bar{u}^{(m)}=\left(u_{1}^{(m)}, \ldots, u_{n}^{(m)}\right)$.

\section{The theory CODF}

We will consider $\mathcal{L}:=\left\{+, \cdot,-,{ }^{-1}, 0,1,<^{\prime}\right\}$ the usual language of ordered fields with an extra symbol ' for the derivation. We will call ODF the $\mathcal{L}$-theory whose models are ordered differential fields. From now on, for any ordered differential field $K$ we will denote by $\mathbb{K}$ the $\mathcal{L}$-structure $\left(K,+, \cdot,-,{ }^{-1}, 0,1,<,^{\prime}\right)$.

M. Singer showed in [13] that the $\mathcal{L}$-theory ODF has a model completion and called it the theory of closed ordered differential fields (CODF).

By definition of the model completion, any ordered differential field is a substructure of a model of CODF and CODF is a model complete theory. Hence,

Fact 1.1. If $\mathbb{K}$ is a model of CODF and if a first order $\mathcal{L}$-formula $\phi$ with parameters in $K$ is satisfied in an ordered differential field extension $\mathbb{L}$ of $\mathbb{K}$, then $\mathbb{K}$ satisfies $\phi$.

Furthermore, CODF eliminates quantifiers because it is the model completion of a universal theory (see [12]).

M. Singer also gave an axiomatisation of CODF:

Theorem 1.2 ([13], §2). Let $K$ be an ordered differential field, $K$ is a closed ordered differential field iff

1. $K$ is a real closed field;

2. for any $f, g_{1}, \ldots, g_{m} \in K\{X\}$, such that for all $i \in\{1, \ldots m\}$, $n:=\operatorname{ord}(f) \geq \operatorname{ord}\left(g_{i}\right)$.

If there is $a_{0}, \ldots, a_{n}$ such that $f^{*}\left(a_{0}, \ldots, a_{n}\right)=0, s_{f}^{*}\left(a_{0}, \ldots, a_{n}\right)$ $\neq 0$ and $g_{1}^{*}\left(a_{0}, \ldots, a_{n}\right)>0, \ldots, g_{m}^{*}\left(a_{0}, \ldots, a_{n}\right)>0$, then there is $z \in K$ such that $f(z)=0$ and $g_{1}(z)>0, \ldots, g_{m}(z)>0$.

Corollary 1.3 ([2], Lemma 2.2.). Differential tuples (i.e. tuples of the shape $\left(\bar{u}, \bar{u}^{\prime}, \bar{u}^{\prime \prime}, \ldots, \bar{u}^{(k)}\right)$ ) are dense in $K^{n .(k+1)}$ (regarding to the topology induced by $<$ on $K)$.

\section{Dubois' nullstellensatz for ordered fields}

Our nullstellensatz is inspired by a result on real closed fields originally proved by D. W. Dubois in [3] (the proof can also be found in [9]). However, our proof is much closer to the one given in [1].

In this section, rings and fields are not necessarily differential. For convenience of the reader, before stating the main result, we define radical and real ideals. We also state some well-known results. They can be found in [9] and [1].

Definition 2.1. Let $R$ be a ring. An ideal $I$ of $R$ is said to be real if for any $u_{1}, \ldots, u_{n} \in R$, such that $\sum_{i=1}^{n} u_{i}^{2} \in I, u_{i} \in I$ for all $i$. 
Lemma 2.2 ([1], lemme 4.1.6). Let $R$ be a ring and $I$ be a prime ideal of $R, I$ is real iff the fraction field of $R / I$ is formally real (and so it is possible to define an order on it).

Definition 2.3. Let $R$ be a ring and $I$ an ideal. The radical of $I$ is $\sqrt{I}:=\left\{a \in R: a^{n} \in I\right.$ for some $\left.n \in \mathbb{N}\right\}$.

Definition 2.4. An ideal $I$ in $R$ is said to be radical iff $\sqrt{I}=I$.

Lemma 2.5. The radical of an ideal is a radical ideal.

Lemma 2.6 ([1], lemme 4.1.5). Let $R$ be a ring, if $I$ is a real ideal of $R$, then $I$ is radical (and so $I=\sqrt{I}$ ).

For any ring $A, S_{A}$ denotes the set of sums of squares of $A$.

Definition 2.7. Let $A$ be a ring and $I$ an ideal of $A$, the real radical of $I$ is

$$
\mathcal{R}(I):=\left\{f \in A: f^{2 m}+s \in I \text { for some } m \in \mathbb{N} \text { and } s \in S_{A}\right\} .
$$

Lemma 2.8 ([1], Proposition 4.1.7). Suppose $A$ is a ring and $I$ is an ideal in $A$. The real radical of $I$ is the smallest real ideal containing $I$.

The statement of Dubois' Theorem is the following:

Theorem 2.9. Let $F$ be a real closed field, $I$ an ideal of $F\left[X_{1}, \ldots X_{n}\right]$ and $f \in F\left[X_{1}, \ldots X_{n}\right]$. Suppose that any zero of $I$ in $F^{n}$ is also a zero of $f$. Then $f \in \mathcal{R}(I)$.

\section{Differential ideals}

We recall here well-known results on differential ideals that will be used later. The material of this section can be found in [5] and [8].

Definition 2.10. Let $R$ be a differential ring, an ideal of $R$ is said to be differential iff for any $u \in I, u^{\prime} \in I$.

An ideal in a differential ring will be called real differential if it is real and differential.

Unfortunately, the radical of a differential ideal is not always a differential ideal. But one can show that in a ring containing $\mathbb{Q}$, the radical of a differential ideal is actually differential. More precisely:

Definition 2.11. A Ritt algebra is a differential ring having $\mathbb{Q}$ among its subrings.

For example, any differential field of characteristic zero is a Ritt algebra.

Lemma 2.12 ([5], Lemma 1.8). Let $R$ be a Ritt algebra and $I$ a differential ideal of $R, \sqrt{I}$ is a differential ideal.

Corollary 2.13. Let I be a real (even radical) differential ideal in a Ritt algebra $R, \sqrt{I}=I$. 
Theorem 2.14 (Ritt-Raudenbush). Let $R$ be a Ritt algebra whose radical differential ideals are finitely generated. Then, every radical differential ideal in $R\left\{X_{1}, \ldots, X_{n}\right\}$ is finitely generated.

Proof. See [8], Theorem 1.16.

Theorem 2.15 (Ritt-Raudenbush). Let $R$ be a Ritt algebra whose radical differential ideals are finitely generated. Every radical differential ideal in $R\left\{X_{1}, \ldots, X_{n}\right\}$ is the intersection of a finite number of prime differential ideals.

Proof. See [8], Theorem 1.18.

Note that the hypotheses of these two theorems are fulfilled when $R$ is a differential field.

\section{The nullstellensatz in the differential case}

Here $\mathbb{K}=\left(K,+, .,-,^{-1}, 0,1,<^{\prime}\right)$ is an ordered differential field.

We first need to refine Ritt-Raudenbush Theorem (see 2.15) in the particular case of real ideals.

Lemma 3.1. If $I$ is a real differential ideal in $K\left\{X_{1}, \ldots, X_{n}\right\}$, then $I$ is the intersection of a finite number of prime real differential ideals.

Proof. By 2.15, $I=\bigcap_{i=1}^{m} P_{i}$ where $P_{i}$ are prime differential ideals. W.l.o.g., one may assume that $P_{i}$ are minimal (for the property of being prime and extending $I$ ).

Suppose $P_{1}$ is not real, i.e. there are $u_{1}, \ldots, u_{k} \in K\left\{X_{1}, \ldots, X_{n}\right\} \backslash$ $P_{1}$ s.t. $u_{1}^{2}+\cdots+u_{k}^{2} \in P_{1}$. By the assumption of minimality, for all $i=2, \ldots, m$ there are $v_{i} \in P_{i} \backslash P_{1}$.

Let $v:=\prod_{i=2}^{m} v_{i}$. Clearly, $v \notin P_{1}$ and $v \in \bigcap_{i=2}^{m} P_{i}$.

Then, $\left(u_{1} v\right)^{2}+\cdots+\left(u_{k} v\right)^{2} \in \bigcap_{i=1}^{m} P_{i}=I$. Since $I$ is real, $\left(u_{1} v\right) \in$ $I \subset P_{1}$. Finally, as $P_{1}$ is prime, $u_{1} \in P_{1}$ or $v \in P_{1}$, in both cases, that gives a contradiction.

Definition 3.2. Let $I$ be a prime differential ideal in $K\left\{X_{1}, \ldots, X_{n}\right\}$, $L \supset K$ be a differential extension of $K$ and $\bar{z} \in L$. We will say that $\bar{z}$ is a generic zero of $I$ iff $I=\mathcal{I}(\bar{z}):=\left\{f \in K\left\{X_{1}, \ldots, X_{n}\right\}: f(\bar{z})=0\right\}$.

Lemma 3.3. Let $I$ be a prime differential ideal in $K\left\{X_{1}, \ldots, X_{n}\right\}, I$ is real iff $I$ has a generic zero in some ordered differential extension $L$ of $K$.

Proof. $[\Rightarrow]$ If $I$ is real, then $L:=\operatorname{Frac} K\left\{X_{1}, \ldots, X_{n}\right\} / I$ is formally real and so the order of $K$ can be extended to all $L$.

Consider $\bar{z}:=\bar{X}+I \in L$. Let $f \in K\{\bar{X}\}, f \in I$ iff $f(\bar{X})+I=0$ iff $f(\bar{X}+I)=0$ iff $f(\bar{z})=0$.

$[\Leftarrow]$ If $I$ has a generic zero in an ordered differential extension $L$ of $K$ (say $\left.I=\mathcal{I}(\bar{z}), \bar{z} \in L^{n}\right)$ and $\sum_{i=1}^{n} f_{i}^{2} \in I$ for some $f_{1}, \ldots, f_{n} \in$ $K\left\{X_{1}, \ldots, X_{n}\right\}$. Then $\sum_{i=1}^{n} f_{i}^{2}(\bar{z})=0$. So for all $i, f_{i}(\bar{z})=0$, namely $f_{i} \in I$. 
Remark 3.4. One easily see that if $\bar{z}$ is a generic zero of $I$ and $\bar{z}^{\prime}$ has the same type as $\bar{z}\left(\operatorname{tp}\left(\bar{z}^{\prime} / K\right)=\operatorname{tp}(\bar{z} / K)\right)$, then $\bar{z}^{\prime}$ is also a generic zero of $I$. So, in a $|K|^{+}$-saturated ordered differential extension of $K$, any real differential ideal of $K\left\{X_{1}, \ldots, X_{n}\right\}$ has a generic zero.

Notation. Let $R$ be a differential ring and $a_{1}, \ldots, a_{n} \in R,\left[a_{1}, \ldots, a_{n}\right]$ will denote the differential ideal generated by $a_{1}, \ldots, a_{n}$ in $R$. Similarly, if $E \subset R,[E]$ will denote the differential ideal generated by $E$ in $R$. Moreover, $\left\{a_{1}, \ldots, a_{n}\right\}$ (resp. $\{E\}$ ) will denote the radical differential ideal generated by $a_{1}, \ldots, a_{n}$ in $R$ (resp. by $E$ in $R$ ).

From now on, $K$ is a closed ordered differential field.

Here we write $\mathcal{I}(\mathcal{V}(I))$ for $\mathcal{I}\left(\mathcal{V}_{K}(I)\right)$.

Proposition 3.5. Let $I$ be a differential ideal of $K\left\{X_{1}, \ldots, X_{n}\right\}, I$ is real iff $\mathcal{I}(\mathcal{V}(I))=I$.

Proof. $[\Rightarrow]$ Clearly $\mathcal{I}(\mathcal{V}(I)) \supset I$. For the other inclusion, we first consider a particular case.

If $I$ is prime: By Ritt-Raudenbush Theorem 2.14, there is $g_{1}, \ldots, g_{k}$ $\in K\left\{X_{1}, \ldots, X_{n}\right\}$ such that $I=\left\{g_{1}, \ldots, g_{k}\right\}$. Moreover, by Lemma 3.3 and the fact that $I$ is real, there exists $\bar{z}$ in some ordered differential extension $L$ of $K$ s.t. $I=\mathcal{I}(\bar{z})$.

Let $f \notin I, \mathbb{L}=\exists \bar{X} \bigwedge_{i=1}^{k} g_{i}(\bar{X})=0 \wedge f(\bar{X}) \neq 0$ (because $\bar{z} \in L$ ).

So by fact 1.1, $\mathbb{K} \models \exists \bar{X} \bigwedge_{i=1}^{k} g_{i}(\bar{X})=0 \wedge f(\bar{X}) \neq 0$. Hence, $f \notin \mathcal{I}(\mathcal{V}(I))$.

We showed that $\mathcal{I}(\mathcal{V}(I)) \subset I$

General case: As $I$ is real, by Lemma 3.1, there are prime real differential ideals $P_{i}$ such that $I=\bigcap_{i=1}^{m} P_{i}$. So $\mathcal{I}(\mathcal{V}(I))=$ $\mathcal{I}\left(\mathcal{V}\left(\bigcap_{i=1}^{m} P_{i}\right)\right) \subset \bigcap_{i=1}^{m} \mathcal{I}\left(\mathcal{V}\left(P_{i}\right)\right)=\bigcap_{i=1}^{m} P_{i}=I$.

$[\Leftarrow]$ Suppose $\mathcal{I}\left(\mathcal{V}((I))=I\right.$. Let $f_{1}, \ldots, f_{l} \in K\left\{X_{1}, \ldots, X_{n}\right\}$ such that $f_{1}^{2}+\cdots+f_{l}^{2} \in I, f_{1}^{2}+\cdots+f_{l}^{2} \in \mathcal{I}(\mathcal{V}(I))$. Equivalently, $f_{1}^{2}+\cdots+f_{l}^{2}$ vanishes on $\mathcal{V}(I)$. Therefore $f_{1}, \ldots, f_{l}$ vanish on $\mathcal{V}(I)$ and so $f_{1}, \ldots, f_{l}$ belong to $\mathcal{I}(\mathcal{V}(I))=I$.

Let $I$ be a differential ideal in $K\left\{X_{1}, \ldots, X_{n}\right\}$. We consider the real radical of $I$ defined in section 2 and denoted by $\mathcal{R}(I)$.

In [15], Stengle showed the following lemma (see Proposition 3.3). For the sake of completeness, we reproduce the proof here.

Lemma 3.6. Let I be a differential ideal of $K\left\{X_{1}, \ldots, X_{n}\right\}, \mathcal{R}(I)$ is a real differential ideal.

Proof. By Lemma 2.8, $\mathcal{R}(I)$ is a real ideal. It remains to show that $\mathcal{R}(I)$ is differential.

Let $u \in \mathcal{R}(I)$ i.e. $u^{2 m}+s \in I$ with $s \in S_{K\left\{X_{1}, \ldots, X_{n}\right\}}$. First we compute $\left(u^{m}\right)^{(m)}=z \cdot\left(u^{(1)}\right)^{m}+u \cdot p$ where $p \in K\left\{X_{1}, \ldots, X_{n}\right\}$ and $z$ is a nonzero integer. Second we show that if $v^{2}+s \in I$ and $s \in S_{K\left\{X_{1}, \ldots, X_{n}\right\}}$ then for all $k \in \mathbb{N}, v^{(k)}$ belongs to $\mathcal{R}(I)$. 
- One shows by induction that

$$
\left(u^{m}\right)^{(n)}=\sum z_{\bar{e}} \cdot u^{m-\left(e_{1}+\cdots+e_{n}\right)} \cdot \prod_{j=1}^{n}\left(u^{(j)}\right)^{e_{j}}
$$

where the sum goes over the tuples $\bar{e}:=\left(e_{1}, \ldots, e_{m}\right)$ such that 1. $e_{1}+2 e_{2}+\cdots+n . e_{n}=n$ and $e_{i} \in \mathbb{N}$ and the $z_{\bar{e}}$ are non-zero natural numbers (actually we got an abstract version of Faà di Bruno's Formula).

Now for $n=m$ all terms are multiplied by a power of $u$, excepted if $e_{1}=m$ and $e_{2}=\cdots=e_{m}=0$. In that case we get $z \cdot u^{m-m} \cdot \Pi_{j=1}^{m}\left(u^{(j)}\right)^{e_{j}}=z \cdot u^{0} \cdot\left(u^{(1)}\right)^{m}$ where $z$ is a non-zero natural number.

- Let $k$ be an integer. We will show by induction that if $v^{2}+s \in I$ and $s:=\sum a_{i}^{2} \in S_{R}$ then $v^{(k)}$ belongs to $\mathcal{R}(I)$. Leibniz rule gives

$$
\left(v^{2}\right)^{(2 k)}=\sum_{i=0}^{2 k}\left(\begin{array}{c}
2 k \\
i
\end{array}\right) v^{(i)} v^{(2 k-i)} .
$$

By induction hypothesis, all terms belong to $\mathcal{R}(I)$ excepted maybe $\left(\begin{array}{c}2 k \\ k\end{array}\right) v^{(k)} v^{(k)}(\star)$. Moreover all $a_{i}$ also have property $(\star)$. If $v^{2}+\sum a_{i}^{2} \in I$ then $\left(v^{2}\right)^{(2 k)}+\sum\left(a_{i}^{2}\right)^{(2 k)} \in I$. Hence by $(\star)$,

$$
\left(\begin{array}{c}
2 k \\
k
\end{array}\right)\left(\left(v^{(k)}\right)^{2}+\sum\left(a_{i}^{(k)}\right)^{2}\right) \in \mathcal{R}(I) .
$$

So $v^{(k)} \in \mathcal{R}(\mathcal{R}(I))=\mathcal{R}(I)$.

Now we complete the proof in letting $v:=u^{m}$. Hence $v^{(m)} \in \mathcal{R}(I)$. So $v^{(m)}=\left(u^{m}\right)^{(m)}=z \cdot\left(u^{(1)}\right)^{m}+u \cdot p \in \mathcal{R}(I)$. Then, as $\mathcal{R}(I)$ is radical, $u^{(1)} \in \mathcal{R}(I)$.

Lemma 3.7. Let $I \subset K\left\{X_{1}, \ldots, X_{n}\right\}$ be a differential ideal. The real radical of $I, \mathcal{R}(I)$ is the smallest (i.e. intersection of all) real differential ideal(s) of $K\left\{X_{1}, \ldots, X_{n}\right\}$ containing $I$.

Here $\mathbb{K}$ is still a model of CODF.

Theorem 3.8 (Nullstellensatz). Let I be any differential ideal of $K\left\{X_{1}\right.$, $\left.\ldots, X_{n}\right\}, \mathcal{I}(\mathcal{V}(I))=\mathcal{R}(I)$

Proof. Firstly, as $\mathcal{R}(I)$ is real, by Proposition 3.5, $\mathcal{I}(\mathcal{V}(\mathcal{R}(I)))=\mathcal{R}(I)$.

Moreover, $I \subset \mathcal{R}(I)$, so $\mathcal{I}(\mathcal{V}(I)) \subset \mathcal{I}(\mathcal{V}(\mathcal{R}(I)))=\mathcal{R}(I)$.

Secondly, we have that $\mathcal{I}(\mathcal{V}(I))$ is real and contains $I$. So by Lemma $3.7, \mathcal{R}(I) \subset \mathcal{I}(\mathcal{V}(I))$.

\section{Prime real differential ideals}

The results in this section are adjustments to real differential ideals of some classical results of differential algebra (see for example [5], Chapter II).

For some technical reasons, we will need the following three lemmas: 
Lemma 4.1 ([5], Lemma 1.6). Let $S$ and $T$ be any subsets of a differential ring. Then $\{S\} .\{T\} \subset\{S . T\}$.

Lemma 4.2. Let $I_{1}, I_{2}$ be differential ideals of $K\left\{X_{1}, \ldots, X_{n}\right\}$,

$$
\mathcal{R}\left(I_{1}\right) \cdot \mathcal{R}\left(I_{2}\right) \subset \mathcal{R}\left(\left[I_{1} \cdot I_{2}\right]\right) .
$$

Proof. In this proof $S:=S_{K\left\{X_{1}, \ldots, X_{n}\right\}}$.

Let $h \in \mathcal{R}\left(I_{1}\right) \cdot \mathcal{R}\left(I_{2}\right), h=f . g$ where $f^{2 k}+s_{1} \in I_{1}$ and $g^{2 l}+s_{2} \in$ $I_{2}$ with $k, l \in \mathbb{N}, l \leq k$ and $s_{1}, s_{2} \in S$. Hence, $\left(f^{2 k}+s\right)\left(g^{2 l}+s^{\prime}\right)=$ $f^{2 k} g^{2 l}+\bar{s} \in I_{1} . I_{2}$ (for some $\bar{s} \in S$ ). Therefore, $f^{2 k} g^{2 k}+\bar{s} . g^{2(k-l)} \in$ $\left[I_{1} \cdot I_{2}\right]$. Finally, we get $h^{2 k}+t \in\left[I_{1} \cdot I_{2}\right]$ with $t=\bar{s} \cdot g^{2(k-l)} \in S$.

Lemma 4.3. Consider a multiplicatively closed set $M$ in $A$ and $J$ a proper maximal real differential ideal among the real differential ideals $I$ such that $I \cap M=\varnothing$. Then $J$ is prime.

Proof. Looking for a contradiction, suppose $b_{1}, b_{2} \notin J$ and $b_{1} . b_{2} \in J$. By maximality of $J$, there exists $a_{1}, a_{2} \in M, a_{1} \in \mathcal{R}\left(\left\{J, b_{1}\right\}\right)$ and $a_{2} \in \mathcal{R}\left(\left\{J, b_{2}\right\}\right)$. By Lemma $4.2, a_{1} . a_{2} \in \mathcal{R}\left(\left\{\left\{J, b_{1}\right\} .\left\{J, b_{2}\right\}\right\}\right)$. Now we use Lemma 4.1 and get $a_{1} \cdot a_{2} \in \mathcal{R}(\{J\})=\mathcal{R}(J)=J$. Therefore, as $M$ is multiplicatively closed, $a_{1} \cdot a_{2}$ belongs to both $M$ and $J$. A contradiction.

The proposition below easily follows from Theorem 3.1 and Lemma 3.7. However, we prove it as a consequence of Lemma 4.3 above.

Proposition 4.4. Let I be a proper differential ideal in $K\left\{X_{1}, \ldots, X_{n}\right\}$. The real radical of $I$ is the intersection of all prime real differential ideals of $A$ containing $I$ (if $I$ is not contained in any prime real differential ideal, then $\left.\mathcal{R}(I)=K\left\{X_{1}, \ldots, X_{n}\right\}\right)$.

Proof. Let $a \notin \mathcal{R}(I)$ and $M$ the multiplicatively closed set $\left\{a^{k}: k \in\right.$ $\mathbb{N} \backslash\{0\}\}$. Let $J$ be a proper real differential ideal which is maximal among the ones which contain $I$ and are disjoint to $M$ (by Zorn Lemma, such an ideal does exist). By Lemma 4.3, $J$ is prime and the proof is complete.

We work now in the following situation: let $B:=K\left\{X_{1}, \ldots, X_{n}\right\}$ and $A \subset B$ a differential subring, $P$ a prime differential ideal in $A$ and $I$ a differential ideal in $B$.

Definition 4.5. We will say that $I$ contracts to $P$ iff $I \cap A=P$.

If $I \subset B$ is real and contracts to $P$ in $A$, can we find a prime real differential ideal $J$ of $B$ extending $I$ which contracts to $P$ ? The next proposition gives a positive answer under a suitable hypothesis on $I$.

Proposition 4.6. Let $I$ be a proper real differential ideal in $B$ which contracts to $P$ and such that for all $a \in A$ and $b \in B, a . b \in I$ implies $a \in I$ or $b \in I$. Then $I$ is an intersection of prime real differential ideals also contracting to $P$. 
Proof. Let $x \in B \backslash I$ and $M:=\left\{a \cdot x^{k}: a \in A \backslash P, k \in \mathbb{N}\right\}$ the multiplicatively closed subset of $B$ generated by $A \backslash P$ and $x$. By the hypothesis made on $I, I \cap M=\varnothing$. Consider a real differential ideal $J$ which is maximal among the ones which also contract to $P$ and are disjoint to $M$. By Lemma $4.3, J$ is prime. Moreover, $J$ doesn't contain $x$. Finally, let us show that $J \cap A=P$ : if $a \in J \cap A$, then $a \notin M$, so $a \notin A \backslash P$, therefore $a \in P$.

\section{Positivstellensatz}

If $f \in K\left\{X_{1} \ldots X_{n}\right\}$, then $f^{*}$ will denote an ordinary polynomial such that $f(\bar{X})=f^{*}\left(\bar{X}, \bar{X}^{\prime} \ldots \bar{X}^{(k)}\right)$. Moreover, if $A \subset K^{n .(k+1)}, \operatorname{diff}(A)$ will denote the set of differential tuples in $A$, i.e.

$$
\operatorname{diff}(A)=\left\{\left(\bar{u}, \bar{u}^{\prime}, \ldots, \bar{u}^{(k)}\right) \in A\right\} .
$$

The positivstellensatz is based on Lemma 5.2, a result of real geometry mentioned in [1].

Definition 5.1. Let $A$ be a ring and a subset $P$ of $A$. We say that $P$ is a cone of $A$ iff for all $p_{1}, p_{2} \in P$ and $a \in A$,

- $p_{1}+p_{2} \in P$,

- $p_{1} \cdot p_{2} \in P$,

- $a^{2} \in P$.

Lemma 5.2 ([1], Corollaire 4.4.3). Let $F$ be a real closed field. Let $g_{1}, \ldots, g_{s} \in F\left[X_{1}, \ldots, X_{n}\right]$ and $W:=\left\{\bar{x} \in K^{n}: g_{1}(\bar{x}) \geq 0, \ldots, g_{s}(\bar{x}) \geq\right.$ $0\}$. Let $f \in F\left[X_{1}, \ldots, X_{n}\right]$ and $P$ be the cone of $F\left[X_{1}, \ldots, X_{n}\right]$ generated by $g_{1}, \ldots, g_{s}$.

$$
\forall \bar{x} \in W, f(\bar{x}) \geq 0 \Leftrightarrow \exists m \in \mathbb{N}, g, h \in P: f . g={ }_{W} f^{2 m}+h .
$$

We will use a consequence of the axiomatisation of CODF, Corollary 1.3, to transfer this lemma in differential fields.

Theorem 5.3 (Positivstellensatz). Let $K$ be a closed ordered differential field. Let $g_{1}, \ldots, g_{s} \in K\left\{X_{1}, \ldots, X_{n}\right\}$ and $W:=\left\{\bar{x} \in K^{n}\right.$ : $\left.g_{1}(\bar{x}) \geq 0, \ldots, g_{s}(\bar{x}) \geq 0\right\}$. Let $f \in K\left\{X_{1}, \ldots, X_{n}\right\}$ and $P$ be the cone of $K\left\{X_{1}, \ldots, X_{n}\right\}$ generated by $g_{1}, \ldots, g_{s}$.

Suppose moreover that there exists an open set $O$ such that $O \subset$ $W^{*} \subset \operatorname{cl}(O)$, where $W^{*}:=\left\{\bar{x} \in K^{n . d}: g_{1}^{*}(\bar{x}) \geq 0, \ldots, g_{s}^{*}(\bar{x}) \geq 0\right\}$ and for all $i, d>\operatorname{ord}\left(g_{i}\right)$.

$$
\forall \bar{x} \in W, f(\bar{x}) \geq 0 \Leftrightarrow \exists m \in \mathbb{N}, g, h \in P: f . g={ }_{W} f^{2 m}+h .
$$

Proof. Let $f^{*}$ as before and $P^{*}$ be the cone generated by $g_{1}^{*}, \ldots, g_{s}^{*}$. By Lemma 5.2, one knows that

$\forall \bar{x} \in W^{*}, f^{*}(\bar{x}) \geq 0 \Leftrightarrow \exists m \in \mathbb{N}, g^{*}, h^{*} \in P^{*}: f^{*} \cdot g^{*}=W^{*}\left(f^{*}\right)^{2 m}+h^{*}$.

If $\forall \bar{x} \in W, f(\bar{x}) \geq 0$ then $\forall \bar{x} \in W^{*}, f^{*}(\bar{x}) \geq 0$ because Lemma 1.3 implies that $\operatorname{diff}(O)$ is dense in $W^{*}$. Moreover, the converse is obviously true. 
So, $\forall \bar{x} \in W, f(\bar{x}) \geq 0$ iff $\forall \bar{x} \in W^{*}, f^{*}(\bar{x}) \geq 0$.

Clearly, by 5.2 ,

$\forall \bar{x} \in W^{*}, f^{*}(\bar{x}) \geq 0 \Leftrightarrow \exists m \in \mathbb{N}, g^{*}, h^{*} \in P^{*}: f^{*} \cdot g^{*}=W^{*}\left(f^{*}\right)^{2 m}+h^{*}$.

Finally, using continuity of the polynomials and density of $\operatorname{diff}(O)$, one obtains the following

$$
\begin{gathered}
\exists m \in \mathbb{N}, g^{*}, h^{*} \in P^{*}: f^{*} \cdot g^{*}={ }_{W^{*}}\left(f^{*}\right)^{2 m}+h^{*} \\
\Leftrightarrow \exists m \in \mathbb{N}, g, h \in P: f . g={ }_{W} f^{2 m}+h .
\end{gathered}
$$

As mentioned in the introduction, T. Grill has found a counterexample to an abstract and more general version of the theorem above. This explain the fact that we had to put some conditions on $W^{*}$. Moreover note that in the proof we only use the fact that $\operatorname{diff}\left(W^{*}\right)$ is dense in $W^{*}$ and so this weaker condition would be sufficient to get the result.

\section{A generalisation to ordered fields with several derivations}

We consider here ordered fields equipped with several commuting derivations. We work in the usual language of ordered fields enriched with a set $\Delta:=\left\{\delta_{1}, \ldots, \delta_{m}\right\}$ of $m$ unary function symbols. We will call partial differential ring (resp. field) or $\Delta$-ring (resp. field), a ring (resp. field) equipped with $m$ commuting derivations denoted $\delta_{1}, \ldots, \delta_{m}$ (by commuting, we mean for all $i, j, \delta_{i} \delta_{j}=\delta_{j} \delta_{i}$.

Let $\Theta:=\left\{\delta_{1}^{e_{1}} \ldots \delta_{m}^{e_{m}}: e_{1}, \ldots, e_{m} \in \mathbb{N}\right\}$. We will define an order on the set $\Theta$ of differential operators by saying that $\delta_{n}^{e_{1}} \ldots \delta_{m}^{e_{m}}$ is bigger that $\delta_{n}^{f_{1}} \ldots \delta_{m}^{f_{m}}$ iff $\left(e_{1}+\cdots+e_{m}, e_{1}, \ldots, e_{m}\right)$ is bigger that $\left(f_{1}+\cdots+\right.$ $\left.f_{m}, f_{1}, \ldots, f_{m}\right)$ according to the lexicographical order. We will denote by $\theta_{k}$ the $k$-th element of $\Theta$ (the order types of $\Theta$ and $\omega$ are the same).

Let $K$ be a $\Delta$-field and $n, k$ be positive integers. An element $\bar{x}$ of $K^{n .(k+1)}$ is said to be a differential tuple iff $\bar{x}=\left(\bar{u}, \theta_{1}(\bar{u}), \ldots, \theta_{k}(\bar{u})\right)$ for some $\bar{u} \in K^{n}$.

We will work in the theory $m$-CODF of closed ordered $\Delta$-fields studied and axiomatised by C. Rivière in [10] and M. Tressl in [16]. They showed that $m$-CODF is the model completion of the universal theory of ordered fields endowed with $m$ commuting derivations and so eliminates quantifiers.

In order to generalize in this more general setting the proofs given before, we will use analogues of Ritt-Raudenbush Theorems for partial differential fields mentioned by T. McGrail in [7] (see [6], pp. 126-129, for a proof).

Using these results, one can easily mimic all the proofs leading to Theorem 3.8 and so obtain the following:

Theorem 6.1. Let $K$ be a model of $m$-CODF. Let $I$ be a $\Delta$-ideal of $K\left\{X_{1}, \ldots, X_{n}\right\}, \mathcal{I}(\mathcal{V}(I))=\mathcal{R}(I)$. 
To prove Proposition 4.6 for $\Delta$-fields, we need the following lemma which is an analogue of Lemma 4.1 for $\Delta$-rings. Its proof is exactly similar to the one of Lemma 1.6 in [5] but using Lemma 2.2.9 from [7] instead of Lemma 1.3 from [5].

Lemma 6.2. Let $S$ and $T$ be any subsets of a $\Delta$-ring. Then $\{S\} .\{T\} \subset$ $\{S . T\}$.

We effortlessly obtain the following analogue of Proposition 4.6:

Proposition 6.3. Let $A \subset B=K\left\{X_{1}, \ldots, X_{n}\right\}$ and $P$ is a prime $\Delta$-ideal of $A$. Let $I$ be a proper real $\Delta$-ideal in $B$ which contracts to $P$ and such that for all $a \in A$ and $b \in B, a . b \in I$ implies $a \in I$ or $b \in I$. Then $I$ is an intersection of prime real $\Delta$-ideals also contracting to $P$.

Now, in order to generalize the positivstellensatz proved in section 5 , we need a corollary of $m$-CODF axiomatisation:

Corollary 6.4 (see [11], Lemma A.3.2). Differential tuples are dense in $K^{n \cdot(k+1)}$.

Hence, the proof of Theorem 5.3 is easily adapted to a proof of the following theorem. As before $f^{*}$ is an ordinary polynomial s.t. $f(\bar{u})=f^{*}\left(\bar{u}, \theta_{1}(\bar{u}), \ldots, \theta_{k}(\bar{u})\right)$.

Theorem 6.5. Let $K$ be a closed ordered $\Delta$-field. Let $g_{1}, \ldots, g_{s} \in$ $K\left\{X_{1}, \ldots X_{n}\right\}$ and $W:=\left\{\bar{x} \in K^{n}: g_{1}(\bar{x}) \geq 0, \ldots, g_{s}(\bar{x}) \geq 0\right\}$. Let $f \in K\left\{X_{1}, \ldots X_{n}\right\}$ and $P$ be the cone of $K\left\{X_{1}, \ldots X_{n}\right\}$ generated by $g_{1}, \ldots, g_{s}$.

Suppose moreover that there exists an open set $O$ such that $O \subset$ $W^{*} \subset \operatorname{cl}(O)$, where $W^{*}:=\left\{\bar{x} \in K^{n . d}: g_{1}^{*}(\bar{x}) \geq 0, \ldots, g_{s}^{*}(\bar{x}) \geq 0\right\}$ and $d$ is such that for all $i=1, \ldots, s$, if $\theta_{k}$ appears in $g_{i}$, then $d>k$.

$$
\forall \bar{x} \in W, f(\bar{x}) \geq 0 \Leftrightarrow \exists m \in \mathbb{N}, g, h \in P: f . g={ }_{W} f^{2 m}+h .
$$

\section{Acknowledgments}

I would like to thank my Ph.D. advisor, Françoise Point, for her many useful remarks and devoted help during the preparation of this paper. I thank Marcus Tressl as well for communicating to me Stengle's paper on differential inequalities and Gill's counterexample. I'm also grateful to Nikesh Solanki who asked me for the case of fields with several derivations.

\section{References}

[1] J. Bochnak, M. Coste, M.-F. Roy, Géométrie algébrique réelle, Springer-Verlag, (1987).

[2] T. Brihaye, C. Michaux, C. Rivière, Cell decomposition and dimension function in the theory of closed ordered differential fields, Ann. Pure Appl. Logic 159 (2009), no. 1-2, pp. 111-128. 
[3] D. W. Dubois, A nullstellensatz for ordered fields, Arkiv för Matematik, Band 8, nr 13 (1969), pp. 111-114.

[4] T. Grill, Contributions to Differential, Real Algebra and its Connection to Differential Equations, Ph.D. Thesis, Universität Regensburg, 1997.

[5] I. Kaplansky, An introduction to differential algebra, Hermann, 1957.

[6] E. R. Kolchin, Differential algebra and algebraic groups, Academic Press, New York, 1973

[7] T. McGrail, The Model Theory of Differential Fields with Finitely Many Commuting Derivations, The Journal of Symbolic Logic, Vol. 65, No. 2, (Jun., 2000), pp. 885-913.

[8] D. Marker, M. Messmer, A. Pillay, Model theory of fields, second edition, AK Peters, Ltd., 2006.

[9] A. Prestel, Lectures on Formally Real Fields, Springer no. 1093, 1984.

[10] C. Rivière, The theory of closed ordered differential fields with $m$ commuting derivations, C. R. Acad. Sci. Paris, Ser. I 343 (2006).

[11] C. Rivière, Model companion of theories of differential fields, Differential cell decomposition in closed ordered differential fields, Ph.D. Thesis, Université de Mons-Hainaut, 2005.

[12] G. E. Sacks, Saturated Model theory, second edition, World Scientific, 2010.

[13] M. Singer, The model theory of ordered differential fields, Journal of Symbolic Logic 43 (1978), no. 1, 82-91.

[14] G. Stengle, A Nullstellensatz and a Positivstellensatz in Semialgebraic Geometry, Math. Ann. 207 (1974), pp. 87-97.

[15] G. Stengle, Algebraic theory of differential inequalities, communications in algebra, 19(6), 1743-1763 (1991).

[16] M. Tressl, The uniform companion for large differential fields of characteristic zero, Trans. Amer. Soc. 357 (2005) 3933-3951. 